\title{
Visión histórica de la Sociedad Chilena de Enfermedades Respiratorias en sus 85 años ${ }^{\ddagger}$
}

\author{
MANUEL BARROS M.*
}

\section{Historic sight of the Chilean Society of Respiratory Diseases in its $85^{\text {th }}$ anniversary}

The Chilean Society of Respiratory Diseases was founded in 1930. It was initially named as "Society of Tisiology" since tuberculosis was a major health problem at that time. From then on, its development has mirrored the changes that Chilean society and medical sciences have undergone during XX and XXI centuries. Nowadays, the Chilean Society of Respiratory Diseases embraces distinct disciplines and types of health professionals, maintaining its involvement in clinical and public health issues. This review mentions the pioneers of Respiratory Medicine in Chile, as well as some of the most relevant actors, institutions and activities that have been developed by our society during its 85 years of existence.

Key words: History of Medicine; Chilean Respiratory Medicine.

\section{Resumen}

La Sociedad Chilena de Enfermedades Respiratorias fue fundada en 1930. Inicialmente fue denominada "Sociedad Chilena de Tisiología" ya que en esa época la Tuberculosis era un problema crucial de salud. Desde entonces el desarrollo de la Sociedad ha reflejado los cambios que han experimentado la sociedad chilena y las ciencias médicas en el siglo XXY en los inicios del siglo XXI. Actualmente la Sociedad Chilena de Enfermedades Respiratorias abarca diversas disciplinas y profesionales de la salud, manteniendo su compromiso con temas clínicos y de salud pública. Este artículo menciona a los fundadores de la Medicina Respiratoria en Chile, como también a los actores, instituciones y actividades más relevantes que se han desarrollado en nuestra Sociedad durante sus 85 años de existencia.

Palabras clave: Historia de la Medicina; Medicina Respiratoria Chilena.

La historia de la Sociedad Chilena de Enfermedades Respiratorias (SER) desde su nacimiento en 1930 hasta nuestros días refleja los cambios que han ocurrido en la Medicina Chilena a lo largo del Siglo XX e inicios del Siglo XXI.

La Sociedad Chilena de Enfermedades Respiratorias tiene una característica que la distingue: acoger a todos aquellos profesionales interesados en el estudio de las Enfermedades Respiratorias y en la solución de los problemas que éstas producen en las personas. Integra diferentes disciplinas como medicina clínica y salud pública, distintos profesionales (kinesiólogos, enfermeras, tecnólogos médicos) e incluye médicos especialistas en medicina respiratoria en diferentes etapas de la vida: neonatal, pediátrica, de adultos y ancianos. Por ello, el quehacer de los miembros de la SER se ha desarrollado en la mejoría de los cuidados de los pacientes en todos los niveles de atención y también se han involucrado en la prevención de problemas de salud y en la creación de programas de salud, guías clínicas y más recientemente en los protocolos de atención de las Garantías Explícitas de Salud (GES).

* Profesor Adjunto, Escuela de Medicina. Facultad de Medicina, Universidad de Valparaíso.

Unidad de Enfermedades Respiratorias, Servicio de Medicina Interna, Hospital Carlos Van Buren, Valparaíso.

‡ Texto basado en la conferencia pronunciada en las $34^{\text {as }}$ Jornadas de Otoño de la Sociedad Chilena de Enfermedades Respiratorias, Olmué, V Región. 28 de marzo de 2015. 
Al revisar la historia de la SER nos podemos dar cuenta que desde sus inicios como Sociedad Chilena de Tisiología, estaba presente este abordaje multidimensional de los problemas respiratorios, involucrando aspectos clínicos y una visión de salud pública ${ }^{1}$.

Es así como muchos miembros de nuestra Sociedad desarrollaron posteriormente su vocación asumiendo responsabilidades en Programas nacionales, y en la actividad política.

La creación de la SER, por un grupo de médicos visionarios como Sociedad Chilena de Tisiología en 1930, tuvo como objetivo unir a diferentes personas en el manejo y tratamiento de la Tuberculosis pulmonar. En esa época, esta enfermedad no tenía aún tratamiento eficaz en Chile y era el principal problema de salud pública, tanto por su elevada morbilidad como por constituir la principal causa de muerte en nuestro país, con una tasa de mortalidad mayor a 250 por 100.000 habitantes.

En 1924 se habían creado la Caja del Seguro Obrero Obligatorio y la Caja de Previsión de Empleados Particulares, que representaron un avance muy significativo en la constitución de un sistema de previsión social en Chile.

El mismo año se creó el Ministerio de Higiene, Asistencia y Previsión Social que agrupó diferentes iniciativas estatales en estas tres materias tomando tanto el cuidado de la salud como las condiciones de vida y trabajo de los sectores más desfavorecidos de la población. La labor central de este ministerio estuvo enfocada a "los dos problemas más graves de nuestro país: la organización de la Sanidad y de la Asistencia Social y la organización del Trabajo y de la Previsión Social"2. Como parte de su labor incluyó la creación de un boletín en que se divulgaban los problemas sociales de mayor gravedad, y educaba a la población sobre cambios conductuales que permitirían reducirlos, pero también realizó un abordaje multidisciplinario que incluía la prevención, separando a los niños de padres enfermos y mejorías en condiciones de habitación, salarios y preocupación por la alimentación y el alcoholismo.

Estas innovaciones se sumaban a la esforzada lucha que se realizaba en el país contra la tuberculosis desde el siglo XIX. La prevalencia de la enfermedad era abrumadora, en 1917 el 96\% de las autopsias presentaban signos de tuberculosis.

A pesar de estas iniciativas, no se observaba mayor impacto en la llamada "peste blanca". Los pacientes con tuberculosis llenaban los servicios de hospitalizados adultos, y eran diagnosticados en etapas avanzadas de la enfermedad lo que se reflejaba en la persistencia de una alta mortalidad causada por esta enfermedad: 269/100.000 habitantes en 1914 y 253/100.000 habitantes en 1926. Mientras tanto en países europeos se observaba una reducción progresiva de la mortalidad en la misma época. Por ejemplo, en Inglaterra estas cifras disminuyeron desde 136 a 89/100.000 habitantes entre 1914 y $1929^{2}$.

En este escenario, fue providencial que el Dr. Héctor Orrego Puelma ${ }^{1,2}$, quien había sido comisionado en 1928 para estudiar Farmacología y Terapéutica en Europa, solicitara cambiar su orientación a Tisiología luego de asistir por invitación a la Cátedra del Profesor Emile Sergeant, como lo relata en forma muy amena el Dr. V Farga ${ }^{1}$. Su solicitud fue aceptada, y luego de regresar a Chile en 1929 dio comienzo a una intensa y fecunda labor, entre la que se cuenta la fundación de la Sociedad Chilena de Tisiología. $\mathrm{Su}$ gran entusiasmo le permitió incorporar los conocimientos adquiridos en Francia al cuidado de los enfermos, creando una Clínica de Tisiología en el Hospital San José, en ese entonces un lazareto para enfermos moribundos. No sólo lo transformó en un Servicio Clínico, sino que logró que el Hospital San José fuera dedicado exclusivamente para la atención de pacientes con tuberculosis, entusiasmando a médicos jóvenes para que se dedicaran a esta nueva especialidad.

Replicando el modelo observado en Europa, creó los servicios de radiología y anatomía patológica, obtuvo fondos también para una Sección de Cirugía y logró que la Casa de Salud de San José de Maipo fuese destinada a tuberculosis, habilitando simultáneamente con el Pensionado, el Pabellón Popular del establecimiento ${ }^{4}$.

La fundación de la Sociedad, y los avances en el manejo de los pacientes con tuberculosis fueron realizados junto con su gran amigo y aliado, el Dr. Sótero del Río Gundián ${ }^{3}$. El Dr. del Río poco después de graduarse como médico viaja a estudiar a Europa. Regresa a Chile en 1926, pero poco tiempo después sufre una tuberculosis pulmonar bilateral que lo hace regresar a Suiza, para efectuarse una "cura de altura". Allá trabaja en un sanatorio, adquiere experiencia como $\mathrm{Ti}$ siólogo y al regresar a Chile en 1929 asume la jefatura de Tisiología de la Dirección General de Beneficiencia. Posteriormente asumió la jefatura de la Sección Broncopulmonar, en el Hospital del Salvador y desde el mismo año preside la recién creada Sociedad Constructora de Establecimientos Hospitalarios, donde impulsa la construcción de modernos establecimientos. El de mayor tamaño, al sur de Santiago y con 360 camas, fue llamado Sanatorio El Peral. Después sería 
ampliado y se denominaría Hospital-Sanatorio El Peral (actual Hospital Sótero del Río). Pero el ambicioso programa desarrollado incluyó también la edificación de Hospitales-Sanatorios similares en Valparaíso y Putaendo. Desde 1934 es Director General de Beneficiencia y Asistencia Social y en 1937 realiza una propuesta muy innovadora para la época: agregar a mediciones de morbilidad y mortalidad una estimación de años de vida útil perdidos por la Tuberculosis. En opinión de M. Laborde "Cruzó tres décadas prestando servicios públicos", ya que desarrolló una destacada carrera política, ocupando el cargo de Ministro de Salubridad varias veces, participó en la Comisión para construir la nueva Facultad de Medicina de la Universidad de Chile (1948) y luego como ministro del interior en 1960, debió encargarse de la política sanitaria tras el sismo de ese año.

La Caja del Seguro Obligatorio ${ }^{6}$ fue la institución que tuvo un rol decisivo en la lucha antituberculosa, desarrollando un modelo médico que incluía la salud no sólo como un concepto biológico, sino también como un derecho social, que permitió disponer de recursos para la atención de enfermos, pero también para desarrollar planes preventivos, orientados a mejorar las condiciones sociales de las personas. Este plan financiado por el Congreso Nacional para tratar enfermedades como la tuberculosis, en opinión del Dr. Orrego Puelma significó una verdadera revolución en la historia de los cuidados sanitarios en Chile. El abordaje utilizado consideraba la complejidad del problema, planteando un diseño científico que recogía la experiencia y conocimiento de países más desarrollados, e incluyó la construcción de sanatorios y dispensarios con diferentes características para permitir una atención progresiva y tuvo la visión de desarrollarse en varias regiones del país. Antes de esta ley los cuidados de salud estaban distribuidos entre diferentes organizaciones públicas y privadas de carácter voluntario y altruista.

Desde la década del 30' se observó esta transformación de los cuidados sanitarios en Chile desde una orientación basada en fundaciones a una política de estado que centralizó los esfuerzos y generó centros de referencia en diferentes zonas del país. Los cambios en las condiciones ambientales y de saneamiento junto con el progreso de los cuidados de salud de la población con una orientación de salud pública y mejoría de los cuidados hospitalarios permitieron en relativamente poco tiempo modificar la situación de morbilidad y mortalidad de la población.

Durante esta década en las sesiones de la So- ciedad de Tisiología se discutieron no solamente los problemas de manejo y mejores opciones de tratamiento de la Tuberculosis, sino que se incluyeron sesiones, trabajos y publicaciones en la revista de la Sociedad sobre una enfermedad emergente en la época, la silicosis. Estos trabajos de investigación abarcaron aspectos clínicos, radiológicos y de salud pública. En 1933, R. Lorca y R. Hevia presentaron en sesión de la Sociedad de Tisiología ${ }^{7}$ un estudio realizado en trabajadores de las cristalerías de Santiago que causó gran impacto por la alta prevalencia de enfermedad que hasta ese momento se confundía con tuberculosis. La Sociedad de Tisiología se convierte en el lugar en el cual se discuten las posibles respuestas médicas, sociales y legales a la enfermedad y gran cantidad de trabajos se publican en la primera revista de la Sociedad, fundada por el Dr. Orrego en $1935^{7-9}$

La silicosis se establece como la enfermedad profesional más grave que afectaba a la población trabajadora del país ${ }^{10,11}$, y constituía una seria amenaza para la vida de los trabajadores mineros, un sector clave para la economía, por lo que era un problema nacional de gran envergadura.

En 1940 el Dr. M. de Viado fue enviado por el Ministerio de Salubridad a la mina de cobre Potrerillos, que tenía las mejores condiciones de prevención de la enfermedad en Chile. En su informe describe que la prevalencia de silicosis era de 30\%. En 1941 se realizó una reunión extraordinaria de la Sociedad Chilena de Tisiología ${ }^{7}$, para discutir este grave flagelo. Entre otros, se presentó el trabajo "Consideraciones sobre una nueva clasificación Nacional en la reparación médico-legal de la Silicosis" presentado posteriormente en la XI Conferencia Sanitaria Panamericana $^{12}$ y Clínica de la Silicosis Pulmonar ${ }^{13}$. Sin embargo, a pesar de los avances en métodos de diagnóstico y prevención, se estimaba en 1955 que todavía existían entre 5 y 8 mil trabajadores enfermos?.

La aprobación de la ley de Medicina Preventiva en 1938 contribuyó en gran medida al cambio de paradigma, y logró que los pacientes con tuberculosis y silicosis fueran diagnosticados más precozmente, y por ello tuviesen un mejor pronóstico.

El Hospital Sanatorio El Peral, inaugurado ese mismo año, fue un establecimiento que dirigido por el Dr. René García Valenzuela, no sólo mejoró el cuidado de los pacientes sino que se transformó en una fuente de investigación en la eficacia de las terapias.

Como fue mencionado previamente en 1935 el Dr. Orrego creó la primera revista chilena de- 
dicada a Enfermedades Respiratorios, "Aparato Respiratorio y Tuberculosis", publicada entre ese año y 1957, fecha en que se fusiona con el "Boletín del Hospital Sanatorio El Peral" (1941-1954) creándose la "Revista Enfermedades del Tórax y Tuberculosis", que se publicó hasta 1965.

En la década de 1950 hay importantes cambios en la estructura sanitaria de Chile y también comienza el desarrollo moderno de nuestra especialidad. Esto último queda reflejado en los sucesivos cambios de nombre de la Sociedad: en 1951 la Sociedad de Tisiología, considerando que el ámbito de acción que tenía se había expandido, se transforma en "Sociedad Chilena de Tisiología y Enfermedades Broncopulmonares" y pocos años después, en 1956, en "Sociedad Chilena de Enfermedades del Tórax y Tuberculosis"3.

La mortalidad general entre 1917 y 1955 disminuyó desde 29,4 a 13,5 por 1.000 habitantes, en gran parte por el mejor control de las enfermedades infecto-contagiosas. Aunque los fallecimientos por enfermedades del aparato respiratorio habían presentado un descenso de $46 \%$ en el mismo período, seguían constituyendo la principal causa de muerte en Chile mortalidad por tuberculosis disminuyó de 238 a $70,2 / 100.000$ habitantes, y ocupaba el $9^{\circ}$ lugar entre las causas de muerte en el país.

En 1952 se inaugura el nuevo Hospital Clínico de la Universidad de Chile, José Joaquín Aguirre que reemplaza al antiguo Hospital San Vicente de Paul en la atención de pacientes del área norte de Santiago.

En ese mismo año se reorganiza la atención sanitaria del país con la creación del Servicio Nacional de Salud. Este fusionó las seis entidades que hasta ese momento proveían diferentes aspectos de la atención de salud y también incluyó los servicios médicos que proveían las municipalidades. Se regionalizó la atención con la creación de 13 zonas de salud y se edificaron también modernos hospitales. Dos de ellos son emblemáticos para nuestra especialidad: el Hospital del Tórax y el nuevo edificio para el Hospital San Juan de Dios, ambos inaugurados en 1954.

En el Hospital del Tórax, actual Instituto Nacional del Tórax, se han formado desde esa época la mayoría de los especialistas chilenos, y muchos extranjeros. Desde su origen se ha distinguido por la atención del paciente por sobre otras consideraciones, en un ambiente grato y cálido ${ }^{14}$, así como por la calidad de la docencia que imparte, y se constituyó como centro de referencia nacional para pacientes con patología pulmonar compleja. Su origen fue el Servicio de Enfermedades Broncopulmonares del hospital del
Salvador, dirigido por Dr. Héctor Orrego Puelma, Profesor Titular de la Cátedra de Tisiología de la Facultad de Medicina de la Universidad de Chile, quien asumió la jefatura del nuevo Servicio de Medicina. Su visión permitió que en este hospital se incluyera el Servicio de Cirugía del Tórax junto a la Cátedra respectiva ambas a cargo del Profesor Armando Alonso Vial ${ }^{14}$, así como unidades de Radiología, Anatomía Patológica y Fisiopatología. La dotación inicial de 292 camas daba cuenta del perfil epidemiológico del país en esa época: $35 \%$ camas se destinaban a enfermos de tuberculosis, $31 \%$ camas para patologías respiratorias no tuberculosas y $34 \%$ camas para cirugía de pulmón ${ }^{15}$.

Posteriormente se incorporaron Inmunología y la Unidad de Cuidados Intensivos ${ }^{14}$ y destacó desde sus inicios por la calidad y cantidad de las prestaciones realizadas. Se constituyó no solamente como el centro de formación de especialistas en Enfermedades Respiratorias, sino que ha destacado también por la incontable cantidad de alumnos de pregrado que han realizado prácticas, así como los numerosos profesionales médicos y no médicos que se han perfeccionado allí durante sus más de 60 años de funcionamiento. Hasta la fecha recibe con generosidad y a pesar de las limitaciones por su carga asistencial, a profesionales de todo el país que desean perfeccionar sus conocimientos o conocer nuevas técnicas.

El mismo año (1954) se inaugura también el nuevo Hospital San Juan de Dios. Tendría gran importancia en el desarrollo de la especialidad ya que el Servicio de Medicina Interna, bajo la magistral jefatura del Dr. Rodolfo Armas Cruz, incorporó, entre otros profesionales, a un grupo de jóvenes médicos que dirigido por el Dr. Victorino Farga Cuesta ${ }^{1}$ formaron parte del nuevo Departamento Broncopulmonar. Ellos se hicieron cargo de la atención de los pacientes con gran dedicación y formularon un nuevo concepto en el manejo de la tuberculosis: el tratamiento controlado bajo supervisión directa, que contribuyó al exitoso control de esta enfermedad y es ahora aplicado en todo el mundo.

Posteriormente y luego de ser nombrado Profesor titular de Neumotisiología de la Universidad de Chile en 1966, el Dr. Farga se trasladó al Hospital del Tórax donde asumió la Cátedra que dejó el Profesor Dr. Héctor Orrego Puelma al jubilar. Desarrolló allí una fructífera labor, formando a gran cantidad de médicos que serían posteriormente no sólo destacados especialistas sino también formadores de las siguientes generaciones.

También fue parte de este grupo, en el Hos- 
pital San Juan de Dios, el Dr. Edgardo Carrasco Calderón. Se incorporó recién recibido de médico, y participó con entusiasmo en el cuidado de enfermos con patología respiratoria ${ }^{16}$. Siempre inquieto, gana una beca de estudios para asistir al Massachussets General Hospital, en Boston donde se forma en Enfermedades alérgicas e inmunología, las que aplicaría posteriormente en forma pionera en Chile, formando gran cantidad de médicos. Se traslada al Hospital del Tórax donde liderará en 1974 su transformación en Instituto de Enfermedades Respiratorias y Cirugía Torácica. Organizador de gran cantidad de cursos bajo el alero de esta institución, tuvo también la visión y perseverancia para crear en 1985 la revista "Enfermedades Respiratorias y Cirugía Torácica", que desde 1990 adquiere su nombre actual: "Revista Chilena de Enfermedades Respiratorias" y es el órgano oficial de nuestra Sociedad.

También en la década del '50, bajo la guía del Dr. Edgardo Cruz Mena, se formó en el Hospital Clínico de la Pontificia Universidad Católica de Chile, uno de los más prolíficos grupos de investigación en nuestra especialidad, con gran cantidad de publicaciones en destacadas revistas nacionales y extranjeras. Este grupo integrado inicialmente con los Drs. Santiago Raddatz y Ricardo Ferretti destacó por la aplicación de pruebas de función pulmonar en la clínica y demostró la utilidad que la exploración funcional respiratoria tenía en la evaluación de distintas enfermedades. Fue además impulsor, gracias al empuje del Dr. Raddatz, de la incorporación la Tisiología dentro de la nueva especialidad de enfermedades broncopulmonares, integrándose con el Servicio de Medicina Interna. Ha mantenido hasta la fecha varias líneas de investigación que integran conocimientos básicos y clínicos, incorporó la enseñanza de la fisiopatología respiratoria en pregrado, recibió a numerosos médicos que realizaron pasantías de perfeccionamiento y se sumó al Hospital del Tórax en la formación de especialistas desde 1964. Bajo la edición del Profesor Dr. Edgardo Cruz y del Dr. Rodrigo Moreno este grupo publicó un libro: "Aparato Respiratorio: Fisiología y Clínica", que fue desde su primera edición y hasta la actualidad de gran acogida en la enseñanza de las Enfermedades Respiratorias dentro de Chile y América Latina. Por decisión de sus autores, desde hace algunos años este libro está disponible gratuitamente en la página web de la Escuela de Medicina de la Pontificia Universidad Católica de Chile.

En su excelente revisión sobre los ancestros de la Neumología el Profesor Cruz Mena ${ }^{17}$ describe de forma muy elocuente la transformación de la especialidad de Tisiología en Neumología, consecuencia por una parte del control de la tuberculosis con los nuevos tratamientos antibióticos, lo que disminuyó su importancia relativa, y por otra de la investigación realizada en Estados Unidos en Fisiología Respiratoria, necesaria para permitir el pilotaje de aviones que volaran a gran altura, un arma decisiva en la Segunda Guerra Mundial. Como lo han destacado el Dr. Cruz ${ }^{17}$ y el Dr. Farga $^{1}$, estos investigadores sentaron las bases de los conocimientos actuales de Fisiología y Fisiopatología Respiratoria y uno en particular, el Dr. Julius H. Comroe, Jr. pudo, en una tarea nada fácil, persuadir además a las autoridades para que transmitieran los avances logrados a la sociedad civil una vez terminadas las hostilidades.

Poco después, en 1945, el Dr. Elías Motles instaló en el Hospital El Peral el primer laboratorio de función pulmonar en Chile, al regresar de Estados Unidos. En los años siguientes presentó estudios funcionales en diferentes patologías tanto médicas como quirúrgicas y poco después se desarrollaron laboratorios similares en el Hospital de la Pontificia Universidad Católica de Chile (Dr. E. Cruz), del Salvador (Dr. H. Donoso) y en el recién inaugurado Hospital del Tórax (Dr. E. García) ${ }^{3}$.

El desarrollo de la Neumología Pediátrica ha tenido un profundo impacto en la salud de los niños de nuestro país. Como lo describió de manera magistral el Dr. Armando Díaz ${ }^{18}$ en su conferencia en el Congreso de nuestra Sociedad el año 2011. Los pediatras interesados en las Enfermedades Respiratorias ya habían formado una Rama ad hoc en el seno de la Sociedad Chilena de Pediatría. Se incorporaron a partir de la década de los ' 70 a la Sociedad Chilena de Enfermedades del Tórax y Tuberculosis potenciando las reuniones académicas, los Congresos de la Especialidad y las publicaciones en la revista de la Sociedad. Representan actualmente un tercio de los socios médicos activos y han presidido la Sociedad durante 5 períodos.

La historia de la Neumología pediátrica en Chile está íntimamente ligada a la Dra. Eliana Ceruti Danús ${ }^{19}$, quien luego de recibirse ganó una beca en el Cardiovascular Research Institute, de la Universidad de California en San Francisco dirigido por el Prof. Julius H. Comroe, Jr. Durante su estadía describe la importancia de la termorregulación de los recién nacidos, lo que transformó los cuidados neonatales. A su regreso a Chile se incorpora al que sería el Hospital Roberto del Río, bajo la dirección del Profesor Julio Meneghello. Allí iniciaría las pruebas funcionales en niños, las mediciones de gases arteriales, la 
formación de la primera Unidad de Cuidados para niños más graves -a la cual llegaron los primeros ventiladores mecánicos para niños en el país- y la formación de muchas generaciones de médicos especialistas en Neumología pediátrica siempre junto a sus inseparables colegas y amigos Drs. Armando Díaz Cruz y Carlos Casar Collazo, con quienes formó lo que llamaron "molécula indivisible"19. Tuvo la visión de regular la formación de especialistas, diseñando el currículum de postítulo, y la extraordinaria capacidad de combinar la docencia, el cuidado de los enfermos y escribir numerosas e importantes contribuciones científicas a la literatura médica.

En la década de 1960 destacó otro participante en la guerra contra el bacilo de Koch en nuestro país y América Latina: el Dr. Luis Herrera Malmsten. El Dr. Herrera alcanzó la jefatura de la Sección Tuberculosis del Instituto Bacteriológico, donde investigó los factores que limitaban el control de la tuberculosis en América Latina ${ }^{20}$. Como resultado de estas investigaciones, estandarizó los procedimientos involucrados en la baciloscopia, desde la toma de la muestra hasta el informe bacteriológico, creó la primera red de laboratorios de tuberculosis en Chile y tuvo además el enorme mérito de escribir el primer "Manual de Microscopia de Tuberculosis", que fue publicado por la Oficina Panamericana de la Salud en 1973 y se mantuvo vigente por diez años, constituyendo una guía de gran utilidad en el control de esta enfermedad.

A principios de la década de 1980 hubo una reactivación de la Sociedad, liderada por el Dr. Patricio González. Junto con los Drs. Manuel Oyarzún y Álvaro Undurraga promovieron nuevas actividades científicas, entre ellas las Jornadas de Otoño que cumplieron 34 años este $2015^{21}$. El Dr. González destacó por su dedicación y entusiasmo en todas las actividades que emprendió. En la Sociedad cumplió de manera destacada como director, secretario, presidente y posteriormente como editor de la sección Panorama (crónica) de la Revista Chilena de Enfermedades Respiratorias. En palabras del Dr. J.C. Rodríguez "miró pasar la vida desde el Instituto Nacional del Tórax" ${ }^{21}$ donde llegó recién recibido de médico y donde desarrolló una fecunda labor clínica, docente y administrativa.

El actual nombre de Sociedad Chilena de Enfermedades Respiratorias fue adoptado en 1988, y se realizó una modificación societaria que permitió la creación de las Sociedades Filiales, inicialmente en Valparaíso y Concepción, y más recientemente en la zona sur (que agrupa a los socios de Temuco, Valdivia, Osorno y Puerto
Montt). Todas ellas han mantenido una constante actividad, y han potenciado la imagen de nuestra Sociedad al involucrarse en los problemas respiratorios que se enfrentan en cada región.

El grave problema de la contaminación atmosférica en Santiago y sus consecuencias en la salud de la población preocupó a miembros de la Sociedad desde los años ' $60^{22}$, pero es a partir de 1990 en que algunos miembros de nuestra Sociedad, entre los que destaca el Dr. Manuel Oyarzún Gómez, inician una verdadera cruzada para que se aborde este complejo problema ${ }^{23,24}$. Inicialmente en ámbitos académicos, pero posteriormente también en la difusión a la sociedad en los últimos años la preocupación por los efectos de la contaminación ambiental en la salud de las personas, y específicamente en el sistema respiratorio ha alcanzado amplio consenso. El Dr. Oyarzún es un destacado académico e investigador, y ha ocupado diferentes cargos en su alma mater, la Universidad de Chile. Luego de una sólida formación que incluyó una beca de especialización con el Profesor John A. Clements, destacó en la investigación de Fisiología respiratoria -específicamente surfactante pulmonar- y posteriormente se interesó en los complejos problemas de la investigación de la contaminación ambiental. Pero también es el Editor del órgano oficial de la SER, la Revista Chilena de Enfermedades Respiratorias, que ha cumplido 30 años de publicación continua.

También en los años ' 90 y por iniciativa de un destacado grupo de médicos de España y América Latina se crearon los Congresos Iberoamericanos, que tuvieron como objetivo reunir a todos los investigadores de este origen y que sobresalían por sus publicaciones en la literatura médica. En nuestra Sociedad la Dra. Carmen Lisboa, destacada especialista e investigadora de la Pontificia Universidad Católica de Chile participó con entusiasmo de estos esfuerzos, logrando en conjunto con el Dr. Rodrigo Moreno, que se realizara el $3^{\text {er }}$ Congreso Iberoamericano en Viña del Mar, en 1994. Estos congresos fueron el germen de la Asociación Latinoamericana de Tórax, que fue fundada dos años más tarde, y de la cual la Dra. Lisboa fue su segunda presidente. En 1996 se realizó en Santiago el II Curso de Epidemiología Clínica, iniciativa de la Dra. Sonia Buist con el auspicio de la American Thoracic Society. La realización de este curso, fue fruto del esfuerzo y visión del Dr. Patricio González, que había asistido como alumno a la primera versión realizada en México. Fue organizado en el seno de la Sociedad y tuvo una exitosa convocatoria, reuniendo en Santiago a destacados neumólogos latinoamericanos que se 
entusiasmaron con el abordaje epidemiológico de problemas clínicos.

Las consecuencias del tabaquismo representan actualmente una enorme carga de enfermedad dentro de nuestra especialidad. Por ello se formó, también en los años ' 90 , una comisión especialmente dedicada a estudiar este tema, y realizar actividades de difusión de nuevos conocimientos tanto a la comunidad médica como al público en general. Muchos miembros de la Sociedad han dedicado generosamente gran cantidad de tiempo y esfuerzo, destacando como una de las comisiones más proactivas. Un enorme logro fue integrar en un Comité transversal a profesionales y personas que sin pertenecer a nuestra Sociedad tenían interés en prevenir la gran cantidad de enfermedades causadas por el consumo de tabaco. Este esfuerzo ha dado frutos, logrando modificar la actitud de muchas personas frente al consumo de tabaco, y especialmente promoviendo la aprobación de las sucesivas leyes que han restringido el uso de tabaco en los últimos 20 años.

Especial mención merece el esfuerzo desplegado durante el año 2012, en que la Sociedad emitió declaraciones de posición frente a diversos aspectos de la tramitación de la ley ${ }^{25}$, y logró que sus representes expusieran en la Comisión de Salud del Senado los sólidos argumentos científicos que demuestran el daño que causa el tabaco ambiental. Además consiguió que se emitieran notas en la prensa nacional audiovisual y escrita destacando las evidencias científicas que demostraban las graves consecuencias del tabaquismo y las ventajas de la prohibición de fumar en espacios cerrados como protección de los trabajadores y asistentes no fumadores ${ }^{26}$.

Actualmente tenemos más de 630 socios. El aumento en la membresía, con la incorporación de distintos profesionales que han aportado entusiasmo en la investigación y cuidado de pacientes con Enfermedades Respiratorias, ha permitido diversificar las áreas de interés, enriqueciendo las reuniones, Cursos, Jornadas y el Congreso Anual que este año celebrará su $48^{\mathrm{a}}$ versión.

Entre los profesionales médicos, un tercio son pediatras, lo que ha permitido un interesante intercambio de ideas, y colaboración en la gestación de políticas que permitan una transición adecuada para los niños que han crecido con enfermedades crónicas y ahora enfrentan su adolescencia y juventud obligados a cambiar de equipo tratante.

Un tercio de los miembros de nuestra Sociedad son profesionales no médicos, principalmente kinesiólogos. Ellos han aportado su experiencia en el cuidado de los pacientes, han presentado trabajos de investigación y han sido también activos expositores en las Jornadas y Congresos.

Para potenciar la participación de estos profesionales, se crearon ramas que agrupan a kinesiólogos, enfermeras y tecnólogos médicos, las que han permitido gestionar las actividades de mayor interés para cada grupo, definir invitados y las áreas prioritarias de discusión.

Durante estos 85 años de historia se han elegido 41 directivas, con 38 presidentes (Dr. E. Motles 3 períodos, Drs. Orrego Puelma, Corbalán Trumbull y García Suárez 2 períodos cada uno). Veintiún socios han sido distinguidos con el nombramiento de Socios honorarios por los destacados servicios que han entregado, y sólo 7 miembros han sido nombrados Maestros de la Especialidad, la más alta distinción que otorga nuestra Sociedad:

Dr. Luis Herrera Malmsten (1969)

Dr. Héctor Orrego Puelma (1983)

Dr. Victorino Farga Cuesta (1993)

Dr. Edgardo Cruz Mena (2001)

Dra. Eliana Ceruti Danús (2006)

Dr. Edgardo Carrasco Calderón (2009)

Dr. Manuel Oyarzún Gómez (2013)

Los últimos años han encontrado a la Sociedad en un proceso de incremento de las relaciones internacionales. La activa participación en los cursos de Epidemiología Clínica de la American Thoracic Society, y posteriormente la firma de convenios con la Asociación Latinoamericana del Tórax y este último año con la European Respiratory Society han permitido que la membresía de nuestros socios incluya actualmente la de estas dos últimas. Además se ha firmado, más recientemente, un provechoso convenio con la Sociedad Española de Neumología y Cirugía Torácica, que incluye intercambio académico y de investigación. Estos acuerdos representan una gran oportunidad para los miembros de la SER porque permitirán interactuar con los mejores especialistas del mundo en Enfermedades Respiratorias.

\section{Bibliografía}

1.- FARGA V. $80^{\circ}$ Aniversario de la Sociedad Chilena de Enfermedades Respiratorias: de la Tisiología a la Neumología, pasando por la Medicina Interna. Rev Chil Enferm Respir 2011: 27; 31-6.

2.- Boletín Ministerio de Higiene, Asistencia y Previsión Social. Año 1, No 3 páginas 3-6. 1927.

3.- MENA G. El tránsito de la Tisiología a la especialidad actual en Chile. Enferm Respir Cir Torác 1989; 5: 99108. 
4.- DUARTE I. El Boletín del Hospital-Sanatorio El Peral. Datos para la historia del establecimiento. Ars Médica vol $10 \mathrm{~N}^{\circ}$ 10. En: http://escuela.med.puc.cl/publ/ArsMedica/ArsMedica10/IndiceArsMedica10.html

5.- LABORDE M. Medicina Chilena en el Siglo XX. Corporación Farmacéutica Recalcine 2002.

6.- LÓPEZ CAMPILLAY M. De Ciencia, médicos y enfermos en el siglo XX: La Caja del Seguro Obligatorio y la lucha antituberculosa en Chile. Estudios. $\mathrm{N}^{\circ}$ Especial 2012; Mayo: 53-68. Disponible en: http://www. academia.edu/4408642/Ciencia_m\%C3\%A9dicos_y_ enfermos_en_el_siglo_xx_La_Caja_del_Seguro_ Obligatorio_y_la_lucha_antituberculosa_en_Chile.

7.- VERGARA A. Por el derecho a un trabajo sin enfermedad: trabajadores del cobre y silicosis, Potrerillos 1930-1973. Pensamiento Crítico 2002; No 2, p 1-16. Disponible en: www.pensamientocritico.imd.cl

8.- HEVIA R. Algunas consideraciones sobre la silicosis en Chile. Aparato Respiratorio y Tuberculosis 1935; 1: 1.

9.- PUELMA H, CALDERÓN E. Problemas chilenos de salubridad, Aparato Respiratorio y Tuberculosis 1936; 1: 4 .

10.- GARCÍA VALENZUELA R. Caja de seguro y silicosis. Boletín Médico Social 1939: agosto-septiembre.

11.- El problema médico de la silicosis. Boletín del Hospital Sanatorio El Peral 1941: 2; 1 (Abril).

12.- LORCA R, HEVIA R, DE VIADO M, ANSOLA J. Boletín Oficina Panamericana de Salud 1943; noviembre: 976-981. Disponible en: hist.library.paho.org/Spanish/ BOL/v22n11p976.pdf

13.- LORCA R, HEVIA R, RADDATZ S, OYANGUREN H. Clínica de la Silicosis Pulmonar; Revista Aparato Respiratorio y Tuberculosis 1941; 6: 219-41.

14.- CÉSPEDES J. Cincuenta años del Hospital del Tórax.
Rev Chil Enf Respir 2004; 20: 129-30.

15.- QUEZADA R. Al cumplirse 50 años del Instituto Nacional del Tórax. En: www.bibliotecaminsal.cl (Consultado el 30 de junio de 2015).

16.- CALVO M. In memoriam. Profesor Dr. Edgardo Carrasco Calderón (1928-2015). Rev Chil Enferm Respir 2015; 31: 37-8.

17.- CRUZ E. Ancestros de la Neumología. Rev Chil Enferm Respir 2006; 22: 37-44.

18.- DÍAZ A. $80^{\circ}$ Aniversario de la Sociedad Chilena de Enfermedades Respiratorias: Perspectiva de un pediatra. Rev Chil Enferm Respir 2011: 27; 37-40.

19.- PINTO R. Dra. Eliana Ceruti Danús. Nueva Maestra de la Especialidad. Rev Chil Enferm Respir 2007; 23 : 67-74.

20.- HERRERA MALMSTEN L. Estado actual de la microbiología de la Tuberculosis en América Latina. Bol. Oficina Sanitaria Panamericana 1970; abril: 281-7.

21.- CRUZ E, VALDÉS I. Panorama. Rev Chil Enferm Respir 2011; 27: 58-66.

22.- Declaración de la Academia Chilena de Medicina sobre la contaminación atmosférica de Santiago. Rev Med Chile 2012; 140: 1355-6.

23.- OYARZÚN M, VALDIVIA G. Contaminación atmosférica. Declaración de la Academia de Medicina, Instituto de Chile. Rev Chil Enferm Respir 2012; 28: 171-3.

24.- ARANDA C, BELMAR R, BELLO S, MUÑOZ M, SHEPPARD D, OYARZÚN M. El problema de la contaminación atmosférica de Santiago. Enf Respir Cir Torác 1990; 6: 69-78.

25.- CRUZ E, ASTORGA L. Panorama. Rev Chil Enferm Respir 2012; 28: 69-75.

26.- CRUZ E, ASTORGA L. Panorama. Rev Chil Enferm Respir 2012; 28: 159-61.
Correspondencia a:

Dr. Manuel Barros M.

Facultad de Medicina, Universidad de Valparaíso.

Email: manuel.barros@uv.cl 